\title{
Instrumentación de una estrategia de aprendizaje para la enseñanza de los fenómenos de transporte en las carreras de Ingeniería del Instituto Nacional de México mediante el empleo de simuladores digitales
}

\begin{abstract}
Implementing a learning strategy for teaching transport phenomena in the Engineering of the National Institute of Mexico by using digital simulators
\end{abstract}

Juan Alfonso Castañeda Bravo

Centro Interdisciplinario de Investigación y Docencia en Enseñanza Técnica, México

acastaneda@ciidet.edu.mx

Adriana Lugo García

Centro Interdisciplinario de Investigación y Docencia en Enseñanza Técnica, México

alugo@ciidet.edu.mx

\section{Resumen}

Nuestro trabajo consiste en el diseño, construcción e implementación de prácticas de enseñanza universitaria que incluyen desarrollos de las nuevas tecnologías de la información mediante estrategias de aprendizaje adecuadas para lograr un aprendizaje significativo de la transferencia de calor que se estudia en la gran mayoría de las carreras de ingeniería del Sistema Nacional de Educación Superior Tecnológica.

El propósito principal es diseñar una estrategia de aprendizaje y enseñanza de los fenómenos de transporte a través del diseño e implementación de las prácticas y el análisis de la forma en que se utilizan los simuladores en la enseñanza universitaria. Nuestro trabajo va más allá de la simple incorporación de un programa de cómputo, ya que comprende un diseño de prácticas de trabajo basadas en una buena estrategia de aprendizaje, su seguimiento, su utilización en un curso oficial en el plan de estudios, la evaluación del mismo, así como el manual de los profesores que van a utilizarlo en una o más unidades de su curso. 
Para ello, primero se utiliza el lenguaje $\mathrm{C}++$, construyendo la interface correspondiente de acuerdo a estándares internacionales. Posteriormente se implementa la estrategia adecuada para su aplicación en las clases de transferencia de calor, y se proporciona a los profesores para ver la manera adecuada de aplicarlo. Finalmente, se lleva a cabo la evaluación tomando en cuenta tanto a los profesores como a los alumnos y la estrategia empleada.

Palabras Clave: estrategia de aprendizaje, fenómenos, carreras de Ingeniería, simuladores digitales.

\section{Abstract}

Our work involves the design, construction and implementation of university teaching practices including development of new technologies of information through appropriate learning strategies to achieve meaningful learning of heat transfer is studied in most of the races Engineering of the National System of Higher Education Technology.

The main purpose is to design a strategy for learning and teaching of transport phenomena through the design and implementation of practices and analyzing how simulators are used in higher education. Our work goes beyond the simple addition of a computer program, and which comprises a design work practice based on good learning strategy, monitoring, use in a formal course in the curriculum, assessment same as well as the manual for teachers that will be used in one or more units of their course.

To do this, first the $\mathrm{C}++$ language is used, the corresponding interface built according to international standards. Subsequently the right strategy for application in classes heat transfer is implemented, and provides teachers for the proper way to apply it. Finally, we performed the evaluation taking into account both teachers and students and the strategy employed.

Key words: learning strategy, phenomena, engineering careers, digital simulators.

Fecha recepción: Diciembre $2014 \quad$ Fecha aceptación: Julio 2015 


\section{Introducción}

El software educativo es aquel programa o aplicación realizado con la finalidad de utilizarlo como facilitador del proceso de enseñanza y consecuentemente del proceso de aprendizaje. El software educativo es aquel programa educativo y didáctico, el sinónimo para designar genéricamente a los programas para ordenador creados con la finalidad específica de ser utilizados como medio didáctico, es decir, para facilitar los procesos de enseñanza y de aprendizaje (Marqués, 2003:2).

Su historia se remonta a los años veinte, cuando Sydney L. Pressey desarrolla el primer sistema de programación y la primera máquina de enseñar. Así continúa su desarrollo y no fue sino hasta las décadas de los sesenta cuando se unen a la empresa IBM y al Instituto para Estudios Matemáticos en las Ciencias Sociales (IMSSS) de la Universidad de Stanford, dirigida por Patrick Suppes, con el propósito de desarrollar el primer CAI (Computer Aided Instruction) con base en el currículum de la escuela primaria, e implementar su uso en las escuelas de los estados de California y Mississippi en la Unión Americana. Fue la primera vez que los contenidos y la construcción informática se determinaron de acuerdo a la currícula escolar. En las siguientes décadas dichas aplicaciones evolucionaron en los conceptos de CBT (Computer Based Training) y CBI (Computer Based Instruction) que no estaban sustentados solo en dispositivos, sino en programas educativos que funcionan en una plataforma informática.

El concepto de software educativo continuó su desarrollo y en la actualidad, según Marqués, tiene las siguientes características:

a) Los materiales son elaborados con una finalidad didáctica;

b) Utilizan el computador como soporte en el que los alumnos realizan las actividades diversas;

c) Son interactivos, "reaccionan" inmediatamente a las acciones de los estudiantes y permiten un diálogo y un intercambio de información entre el computador (o dispositivo) y los estudiantes;

d) Individualizan el trabajo de los estudiantes, ya que se adaptan al ritmo de trabajo de cada uno y pueden adaptar sus actividades según las actuaciones de los alumnos; 
e) Son fáciles de usar. Los conocimientos informáticos necesarios para utilizar la mayoría de estos programas son similares a los conocimientos de electrónica necesarios para usar un video, aunque cada programa tiene unas reglas de funcionamiento que es necesario conocer.

Existen otras clasificaciones o tipologías de software educativo, las cuales se basan en aspectos relacionados con:

- Los medios que los soportan, los que incorporan multimedios, aquellos que están basados en el paradigma hipertexto, y lo que hacen uso de la realidad virtual.

- Los objetivos educativos que pretenden facilitar el aprendizaje de conceptos, procedimientos y/o actitudes.

- Las actividades cognitivas que activa: es decir, pueden promover la observación, la memorización, el control psicomotriz, la comprensión, la interpretación, la comparación, el análisis, síntesis, estar basados en resolución de problemas, como medio de expresión para la creación y la experimentación.

- Las bases psicopedagógicas que los sustentan y orientan sobre el aprendizaje: basados en el conductismo, el cognitivismo o el constructivismo.

- La función en la estrategia didáctica: se utilizan para entrenar, para instruir, informar, motivar, explorar, experimentar, evaluar, entretener, etcétera.

- $\quad$ El diseño: centrado en el aprendizaje, centrado en la enseñanza, centrado en el estudiante, proveedor de recursos, entre otros.

En resumen, podemos decir que software educativo es un programa informático que se emplea para educar al usuario. Esto quiere decir que el software educativo es una herramienta tecnológica que tiene una función pedagógica o de enseñanza que por sus características ayuda a la adquisición de nuevos conocimientos y al desarrollo de habilidades.

Existen diferentes clases de software educativo. Algunos de estos programas son diseñados como apoyo al docente. De esta manera, el maestro o el profesor acuden al software para ofrecer sus lecciones o para reforzar una clase. Otros tipos de software educativo, en cambio, se orientan 
directamente al alumno, ofreciéndole un entorno real o virtual en el cual puede aprender por su propia cuenta.

El software educativo es muy importante en la educación a distancia pero también en la presencial porque estas herramientas tecnológicas permiten simular las condiciones que existen en un aula o en la industria en donde el alumno se incorporará al concluir su estancia durante su formación escolar. Así, el estudiante puede "ingresar" a un salón o a una industria de manera virtual, interactuar con el docente a través de videoconferencias, chat o correo electrónico, completar evaluaciones, etcétera.

Es importante destacar que, al igual que los libros de texto se clasifican según las edades, el software educativo también apunta a segmentos específicos de estudiantes. En el proyecto que hoy nos ocupa y de acuerdo a la necesidad de despertar en el estudiante la curiosidad científica, privilegiamos el software cuya función principal es la simulación, que lo lleve a la concreción y conocimiento de situaciones experimentales simuladas, que le permitan aprender y resolver situaciones reales que enfrentará en el desempeño de su especialidad pero con un sustento teórico.

A partir de la experiencia y los resultados obtenidos por los docentes tras usar estos programas, se eligió el software educativo, porque se observó que tiene una función motivadora e innovadora en el estudiante, sobre todo para llamar su atención con herramientas "nuevas". En segundo plano, pero no menos importante, está la función instructiva de regulación del aprendizaje de los estudiantes. Con respecto a los software libres, se puede afirmar que es poco significativo el uso que hacen de ellos.

Los simuladores fueron elegidos de acuerdo a la función del software educativo en la estrategia didáctica que se orienta hacia la experimentación y su característica procedimental. Con ellos se pretende proporcionar información de conceptos básicos y construir en general conocimientos, así como aplicar estos a nuevos contextos a los que, por diversas razones, el estudiante no puede acceder desde el contexto metodológico (el aula), donde desarrolla su aprendizaje. De hecho, buena parte de la ciencia puntera, de frontera, se basa cada vez más en el paradigma de la simulación (Díaz Barriga, 2010). Mediante los simuladores se pueden desarrollar experimentos de ingeniería en el laboratorio de informática con mayor seguridad, es decir, simular situaciones de riesgo sin ningún 
peligro real; así cuando el estudiante llegue a la industria estará preparado para resolver contingencias.

Convencidos de ello retomamos las características principales de los simuladores aplicados en la educación. Para el autor Marqués estos deben:

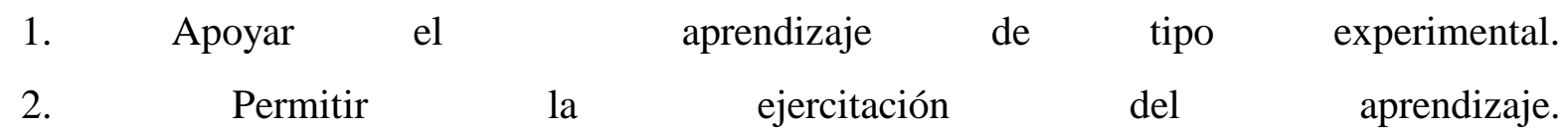

3. Suministrar un entorno de aprendizaje abierto basado en modelos reales. 4. Proporcionar un alto nivel de interactividad.

5. Promover situaciones excitantes o entretenidas que sirvan de contexto al aprendizaje de un determinado tema.

6. Apoyar la naturaleza activa del usuario, quien se convierte en el constructor de su aprendizaje a partir de su propia experiencia.

El presente proyecto tiene que ver con el uso de nuevas tecnologías como una alternativa más para resolver el reto en la formación de alumnos sobre las operaciones de transferencia de calor en Ingeniería, en particular cuando no se cuenta con equipamiento de laboratorio. Lo anterior, por supuesto, sin dejar de ser una excelente introducción para una serie de experiencias de cátedra, con el apoyo de una computadora y un programa de simulación interactiva de un laboratorio.

Asimismo puede ser una modalidad para el Aprendizaje a Distancia, ya que es tan efectivo como las formas tradicionales de educación presencial, cuando se utilizan los métodos y tecnologías adecuadas, esto es, cuando existe interacción entre los profesores-alumnos y cuando disponen de asesoría oportuna de parte del facilitador (Rivera Gallegos, 2007).

En este proyecto se busca la aplicación didáctica de juegos de simulación en el ámbito universitario como herramienta para el proceso enseñanza-aprendizaje en la Ingeniería, particularmente en los fenómenos de transferencia de calor.

Se busca el carácter multidisciplinar de la simulación, unido al creciente interés de los estudiantes por las nuevas tecnologías y la manera como estas influyen positivamente en su motivación y pro- 
actividad en el proceso de enseñanza-aprendizaje y, por lo tanto, en la puesta en práctica de los conocimientos teóricos adquiridos por estos en el aula.

El objetico es incrementar las habilidades y/o competencias mediante el trabajo en grupo e individual.

\section{Antecedentes}

Todas las carreras de Ingeniería Química y otras como Ingeniería de Alimentos, de Recursos Naturales, Mecánica, Nanotecnología, etcétera, estudian en su tronco común los fenómenos de transporte, que constituyen la base profesional de estas carreras, y para el estudio de estas se requiere la aplicación de ecuaciones diferenciales.

El estudio de dichos fenómenos de transporte ha sido una materia compleja, ya que en ella se aplican los aprendizajes previos adquiridos en Química, Física, Matemáticas, balance de materiales y otras más, conocimientos que la mayoría de los estudiantes no tienen muy bien cimentados, por lo que es necesario desarrollar estrategias para aprender estas materias.

Sin duda, la enseñanza de los fenómenos de transporte tiene grandes deficiencias, aunado al hecho de que nuestros estudiantes y algunos de nuestros profesores solo aplican las operaciones unitarias de manera mecánica, sin entender realmente el proceso, trabajando así mediante el ensayo y el error, lo cual es un asunto grave en una escuela de ingeniería. Dicha situación se ha estado agravando con la aparición de simuladores como Chemcad, Highsys, etcétera, donde se les da todo ya digerido al alumno y al profesor, quienes solamente tienen que ver qué operación unitaria se aplica y las corrientes que intervienen. Con esto se pueden obtener resultados espectaculares, pero difícilmente nuestros estudiantes y algunos profesores entienden realmente el fenómeno ocurrido. Por eso es necesario contar con una herramienta que nos ayude a entender el fenómeno en sí mismo y poder, entre otras cosas, hacer reingeniería. Curiosamente hace ya algunos años se participó en un curso de Desarrollo Sustentable en el Instituto Tecnológico de Oaxaca, donde para poder modificar un proceso, lo que curiosamente se requería sobre todo era conocer realmente el proceso, lo cual pudieron constatar algunos de mis compañeros de equipo en dicho curso. 
Encontramos, sobre todo en Internet, muchos "applets" que por lo general se emplean erróneamente o sin guía, de modo que no propician el aprendizaje y se convierten en meros "juguetes“.

Lo anterior lo podemos asegurar después de más de catorce años de experiencia docente en el área de los planteles tecnológicos.

Es necesario desarrollar en los estudiantes la habilidad para el manejo de los fenómenos de transferencia de calor que se aplican principalmente en las ecuaciones de balance. Lo anterior llevado a cabo en solo dos cursos provoca que nuestros estudiantes se conviertan en simples "aplicadores" de fórmulas en situaciones que la mayoría de las veces no les resultan muy claras. La falta de práctica y de una visualización del fenómeno, se puede resolver mediante el uso de simuladores.

Para subsanar lo anterior es necesario darle al estudiante herramientas de apoyo que le permitan aplicar los conocimientos y al mismo tiempo desarrollar estrategias para su mejor utilización y, por lo tanto, lograr un conocimiento profundo de la materia que le permitirá afrontar con éxito las operaciones unitarias.

De acuerdo a una aproximación empírica observacional, se ha encontrado que los índices de reprobación de los estudiantes son muy elevados y que además los contenidos de la materia no se estudian en su totalidad.

Para resolver la problemática señalada se emplean actualmente simuladores digitales, sin embargo, la gran mayoría son programas comerciales generales con propósitos diferentes y orientados hacia las operaciones unitarias, por eso en nuestro trabajo buscamos desarrollar un simulador para la etapa previa a la operaciones unitarias en donde el estudiante aprenda los fundamentos y en sus cursos posteriores los pueda aplicar con éxito.

Sin embargo, la sola herramienta no garantiza el éxito, por lo que debe ir acompañada de una estrategia de aplicación con una metodología bien planeada basada en una estrategia de aprendizaje adecuada, llevar a cabo una evaluación procedente (NO CALIFICACIÓN) y sobre todo, capacitar a los profesores de la materia para que puedan no solo conocer el simulador sino también acompañar a los alumnos. 
Así nuestro proyecto deberá primero desarrollar el programa de aplicación y después implementar una estrategia para su aplicación, evaluando la herramienta en cuanto a su aplicación; además, se deberá dar seguimiento a los resultados en cuanto al impacto académico, lo cual implica que en una segunda etapa, después de elaborar el manual del usuario, se desarrolle un manual para el profesor y se le capacite para obtener los mejores resultados mediante una estrategia de aprendizaje adecuada.

\section{Marco teórico}

Desde el punto de vista didáctico, situamos al estudiante como eje del proceso de enseñanza aprendizaje, afín con el paradigma constructivista del aprendizaje, apoyados en las teorías de Bruner (aprendizaje por descubrimiento) y Ausubel (aprendizaje significativo) (Ferro Fernández, 2010).

Bruner en Díaz Barriga (2010) propone que el aprendizaje debe ser percibido por el alumno como un conjunto de problemas y lagunas por resolver. Para que el aprendizaje sea realmente significativo e importante el estudiante debe formar parte activa del proceso de adquisición del conocimiento y no ser un simple receptor del mismo. Por su parte, Ausubel [2] plantea que el aprendizaje es significativo en la medida que se genere en un ambiente y condiciones que permitan su relación con toda la experiencia anterior del estudiante.

Basados en el esquema metodológico constructivista se plantean estrategias de aprendizaje, como el aprendizaje basado en problemas, el aprendizaje cooperativo y el método de los casos.

"En el aprendizaje basado en problemas, la cuestión fundamental (desde el punto de vista práctico) radica en que los estudiantes descubran lo que necesitan aprender para enfrentar el problema planteado. Los alumnos deben saber diagnosticar un problema a partir de los hechos o los datos duros y no solamente resolver uno ya planteado. En este sentido difiere del llamado aprendizaje basado en temas, donde el profesor establece lo que los estudiantes deben saber y la función de estos últimos es aprenderlo para finalmente aplicarlo a la solución de problemas que les son asignados" (Arias Aranda D., 2009, p. 718). 
El paradigma del aprendizaje cooperativo supera el sistema tradicional competitivo de aprendizaje entre los estudiantes y utiliza un régimen de colaboración entre ellos y de trabajo en equipo. El aprendizaje cooperativo, adecuadamente desarrollado, asegura que los estudiantes se involucren consciente, física y emocionalmente en construir sus propios conocimientos y desarrollar las habilidades correspondientes (Arias Aranda D., 2007).

Desde hace ya hace algún tiempo, en la formación de profesionales las ingenierías y otras disciplinas se ha estado utilizando la estrategia del caso de estudio (o estudio de casos) como método de trabajo docente.

En la formación de ingenieros resulta evidente que los estudios de casos deben orientarse al análisis de procesos concretos de la industria. Así, los estudios de casos deben constituir una explicación detallada y sistemáticamente organizada sobre diferentes procesos de la industria química.

De manera simultánea, en los últimos tiempos se ha reconocido que el estudio sistemático y complejo de las operaciones básicas y de los procesos químico-industriales con una proyección profesional, no puede afrontarse eficazmente sin la utilización de herramientas computacionales adecuadas. De hecho, el desarrollo del concepto de aprendizaje a través de los simuladores y la Simulación de Procesos, como materia de enseñanza, se ha generalizado en las carreras de Ingeniería y otras relacionadas en muchas universidades de todo el mundo.

Los simuladores de proceso están ganando espacio en la docencia universitaria a nivel mundial pues permiten, por un lado, abordar el estudio de procesos complejos a un costo asumible de tiempo y esfuerzo y, por el otro, dotan a los alumnos de experiencia en una herramienta que es ampliamente utilizada en la actividad profesional de los ingenieros.

Podemos señalar que algunas empresas de nuestro país solicitan a los aspirantes que sepan manejar alguno de los simuladores de proceso para su contratación. 
Varios investigadores han señalado los beneficios del uso de los simuladores de proceso en la docencia universitaria de Ingeniería Química. Por ejemplo, Goodyear [14] argumenta que los simuladores permiten al estudiante comprender procesos e instalaciones complejas, que son difícilmente comprensibles aun mediante el contacto directo con la instalación. Kassim y Cadbury insisten en el hecho de que el uso de los simuladores de procesos soporta, refuerza y estimula la independencia cognoscitiva de los estudiantes.

\section{Objetivos}

El objetivo central de este trabajo es desarrollar una estrategia didáctica centrada en el uso de programas de cómputo orientados y su evaluación, con proyección constructivista y cooperativa, para las asignaturas de Transferencia de calor en Ingeniería de los Institutos Tecnológicos.

Se busca introducir el uso de programas de cómputo dirigidos como una herramienta rutinaria de trabajo en todas las asignaturas y actividades de la carrera que requieran de la transferencia de calor.

Sobre todo, se busca desarrollar una estrategia de aprendizaje significativo en el área de transferencia de calor motivando la participación activa de los estudiantes; así como capacitar a los profesores que deseen utilizar dicha herramienta en sus cursos correspondientes.

Finalmente, se busca desarrollar una metodología de evaluación y contrastarla con alumnos que no utilicen estos programas para corroborar su efectividad.

\section{Metas}

- Diseñar e implementar un programa de cómputo aplicando ecuaciones diferenciales para simular los procesos de transferencia de calor durante el aprendizaje de las operaciones unitarias correspondientes.

- $\quad$ Elaborar un manual de usuario del simulador. 
- Capacitar (in situ) sobre todo a los profesores en el uso de esta herramienta.

- Desarrollar una estrategia de aprendizaje mediante el estudio de casos.

- $\quad$ Elaborar el manual correspondiente para el uso de dicha herramienta.

\section{Metodología}

Cambiar la metodología de enseñanza de los fenómenos de transferencia de calor, modificar el empleo de problemas para incrementar la eficiencia del aprendizaje, utilizando modelos desarrollados en la Ingeniería y la interpretación de resultados. Considerar la opinión de los maestros y de los alumnos en relación con las clases teóricas de la asignatura. Cambiar el modelo de evaluación de la misma materia.

Dotar a la asignatura de un carácter eminentemente práctico y aplicado, estudios de casos, sistema de ejemplos y ejercicios utilizados en la asignatura elaborados con esta perspectiva e interpretación de los resultados.

Desarrollar la asignatura con un elevado número de actividades prácticas, sistemáticamente organizadas. Con esto se pretende que los estudiantes tengan suficiente contenido para trabajar individualmente y que no se vean limitados por la asignación de tareas por parte del profesor, mediante la propuesta experimental ya señalada. 


\section{Bibliografía}

Díaz Barriga, F, Hernández Rojas G. (2010). Estrategias Docentes para un aprendizaje significativo. McGraw Hill, 3 a ed.

Rivera Gallegos S. (2007). Uso de simuladores como una alternativa para el quehacer docente en las ciencias experimentales.

Dirección General de Educación Normal y Actualización del Magisterio en el D.F.

V. R. Ferro Fernández, J. O. Gómez Montes, J. F. Palomar Herrero y L. M. Gómez Sainero (2010). Estrategia Didáctica tipo ECTS basada en el uso de simuladores de proceso en la titulación de Ingeniero Técnico Industrial, con especialidad en Química, área de Ingeniería Química, Universidad Autónoma de Madrid.

Arias-Aranda, D. (2007). Simulating Reality for Teaching Strategic Management. Innovations in Education and Teaching International, 44 (3), 273-286.

Geankoplis C.J. (2004). Procesos de transporte y operaciones unitarias, CECSA, $3^{\mathrm{a}}$ ed.

Castañeda, B. J. A. y Lugo G. A. (2014). Revista Iberoamericana para la Investigación y el Desarrollo Educativo, Publicación \# 12, Enero-Junio. RIDE.

Marqués, Pere (2003). El software educativo. Universidad Autónoma de Barcelona. Artículo: http://www.lmi.ub.es/te/any96/marques_software/ Recuperado en:2003-07-02 08:22:33 